University of Nebraska - Lincoln

DigitalCommons@University of Nebraska - Lincoln

April 1972

\title{
Influence of an External Electric Field on the Charge Transfer State of the TCNE-trans-Stilbene Molecular Complex
}

Craig J. Eckhardt

University of Nebraska - Lincoln, ceckhardt1@unl.edu

Follow this and additional works at: https://digitalcommons.unl.edu/chemistryeckhardt

Part of the Chemistry Commons

Eckhardt, Craig J., "Influence of an External Electric Field on the Charge Transfer State of the TCNE-trans-Stilbene Molecular Complex" (1972). Craig J. Eckhardt Publications. 42.

https://digitalcommons.unl.edu/chemistryeckhardt/42

This Article is brought to you for free and open access by the Published Research - Department of Chemistry at DigitalCommons@University of Nebraska - Lincoln. It has been accepted for inclusion in Craig J. Eckhardt Publications by an authorized administrator of DigitalCommons@University of Nebraska - Lincoln. 
charge-induced-dipole interaction. This situation was achieved at $300^{\circ} \mathrm{K}$ by the combination of large ions moving in a gas of low polarizability. It can similarly be achieved at higher temperatures in cases of smaller ions or gases of higher polarizabilities. When the repulsive forces dominate the interaction, the mobilities become very sensitive to the nature of the ion species being studied. In contrast, when the point-chargeinduced-dipole force dominates, the mobilities are mainly determined by the polarizability of the neutral gas and are relatively insensitive to the nature of the ion species.

The mobility theories used in this paper employed some very simple representations of the repulsive forces. Force laws better characterizing the repulsion could likely be deduced from additional mobility data obtained as a function of temperature. In the repulsiondominated regime of interaction, it might also be possible to distinguish measurable differences between the mobilities of positive and negative ions of the same species (e.g., $\mathrm{SF}_{5}^{+}$and $\mathrm{SF}_{5}^{-}$) or perhaps measurable effects on the mobilities caused by inelastic processes occurring in the collisions. For gathering information on molecular collisions, studies of ion transport have the added advantage that many more molecular species exist as ions than are stable as neutrals (e.g., $\mathrm{SF}_{5}$ is not stable but $\mathrm{SF}_{5}-$ is).

\footnotetext{
* Present address: GTE Sylvania Lighting Center, Danvers, Mass. 01923.

${ }^{1}$ A. Dalgarno, M. R. C. McDowell, and A. Williams, Phil. Trans. Roy. Soc. London, Ser. A 250, 411 (1958).

${ }^{2}$ E. W. McDaniel, Collision Phenomena in Ionized Gases (Wiley, New York, 1964), Chap. 9.

3 P. L. Patterson, J. Chem. Phys. 53, 696 (1970).

${ }^{4}$ P. L. Patterson, Bull. Am. Phys. Soc. 14, 610 (1969); 15, 434 (1970); 16, $217(1971)$.

${ }^{5}$ See Ref. 2, Appendix II.

${ }^{6}$ See Ref. 2, p. 433.

7 E. A. Mason and H. W. Schamp, Ann. Phys. (N.Y.) 4, 233 (1958)

${ }^{8} \mathrm{~J}$. O. Hirschfelder, C. F. Curtiss, and R. B. Bird, Molecular Theory of Gases and Liquids (Wiley, New York, 1954), Appendix I-A and pp. 1212-1213.
}

\title{
Influence of an External Electric Field on the Charge Transfer State of the TCNE-trans-Stilbene Molecular Complex
}

\author{
Craig J. Eckhardt \\ Institute for Physical Chemistry, Johannes Gutenberg University, Mainz, Germany and \\ Department of Chemistry, University of Nebraska, Lincoln, Nebraska 68508
}

(Received 20 November 1970)

\begin{abstract}
An experimental investigation of the effect of an external electric field on the absorption spectrum of the tetracyanoethylene-trans-stilbene molecular complex in cyclohexane solution yielded values of $1.1 \pm 0.1 \mathrm{D}$ for the ground state electric dipole moment and $10.5 \pm 0.9 \mathrm{D}$ for the change of the electric dipole moment upon transition to the charge transfer excited state. The ground state electric dipole moment determined by the electro-optic effect is in agreement with the value of $1.4 \pm 0.5 \mathrm{D}$ obtained from the dielectric constant measurements made on solutions of the complex. The directions of the transition moment, the ground state electric dipole moment, and the excited state electric dipole moments were found to be parallel. These results give a $5 \%$ ionic character for the ground state of the complex and $76 \%$ ionic character of the excited state. These results are consistent with the theory of Mulliken.
\end{abstract}

A great deal of data has been obtained on the electronic properties of molecular complexes since the publication of Mulliken's basic papers ${ }^{1}$ on the subject twenty years ago. Most of the data deal with the properties of the ground states of these complexes although there are studies of their luminescence and photochemistry.

The Stark effect can yield valuable information about the excited states of molecules, e.g., the electric dipole moment. Unfortunately such studies are limited to small molecules in the vapor phase. Nevertheless, the study of solution absorption spectra of molecules perturbed by an external electric field offers hope of obtaining information about excited electronic states.

Kuhn, Duhrkop, and Martin ${ }^{2}$ made the first studies of the electrochromic effect. The experimental develop- ments of Labhart ${ }^{3}$ and Czekalla ${ }^{4}$ about ten years ago allowed quantitative treatment of the experimental results. Liptay and his co-workers have extensively developed the theoretical treatment of the effect and clearly related fundamental molecular properties to the experimental quantities. ${ }^{5}$

The electrochromic effect permits determination of the magnitude of the electric dipole moment in the excited state as well as its direction. In favorable cases additional information on the direction of the transition moment and the direction and magnitude of the ground state electric moment can be obtained. With independent determination of the ground state electric moment, the magnitude of the excited state electric moment can always be obtained.

This experimental technique can then be useful in 
determining the characteristic properties of molecular complexes. ${ }^{6}$ These species have ground state electric moments and are characterized by a new electronic absorption band. The moments are described by the Mulliken theory. In particular, the electric dipole moment for a given state permits determination of the total charge distribution of that state. This distribution is in turn described by the wavefunction for the state. Experimental determination of the electric dipole moments therefore allows evaluation of the mixing coefficients for the "no-bond" and dative states of the Mulliken theory. The squares of these coefficients define the percent ionic character for their respective states.

Additional information can be obtained regarding the influence of the field on the transition moment. The size of the cavity the molecular complex occupies in the solvent can also be estimated.

This paper describes the result of electro-optical measurements on the charge transfer absorption band of the molecular complex of tetracyanoethylene (TCNE) with trans-stilbene (hereinafter referred to as stilbene). This research presents an experimental description of the ground state and charge transfer excited state electron density distributions through the electric dipole moments found for the states. Further, the relative directional properties of these moments and the charge transfer transition moment will be discussed. Additional data provided by the electro-optical measurements will also be presented.

\section{THE ELECTROCHROMIC EFFECT}

For an isolated absorption band, the quantity $L_{\chi}{ }^{\prime}\left(\nu_{a}\right)$ can be experimentally obtained:

$$
L_{\chi}{ }^{\prime}\left(\nu_{a}\right)=\left(\epsilon_{F} x-\epsilon_{\mathrm{SOLN}}\right) / F_{a}{ }^{2} \epsilon_{\mathrm{SOLN}},
$$

where $\epsilon_{F} x$ is the extinction coefficient of the solution in a homogenous electric field $\mathbf{F}_{a}$ at wavenumber $\nu_{a}$ and $\epsilon_{\text {SOLN }}$ is the extinction coefficient without the field at the same wavenumber. The linearly polarized light passes through a solution confined between two electrodes such that the propagation vector of the light is perpendicular to the direction of the field with the electric vector of the light at an angle $\chi$ to field direction.

The theoretical expression for $L_{x}{ }^{\prime}\left(\nu_{a}\right)$ has been derived by Liptay ${ }^{7}$ and has the form

$$
\begin{aligned}
& L_{\chi}^{\prime}\left(\nu_{t}\right)=A_{\chi}+(15 h c)^{-1}\left(\frac{d \ln (\epsilon / \nu)}{d \nu}\right)_{\nu a} B_{\chi} \\
& +\left(30 h^{2} c^{2}\right)^{-1}\left[\left(\frac{d \ln (\epsilon / \nu)}{d \nu}\right)_{\nu a}^{2}+\left(\frac{d^{2} \ln (\epsilon / \nu)}{d^{2} \nu}\right)_{\nu a}\right] C_{\chi},
\end{aligned}
$$

where ${ }^{5}$

$$
\begin{aligned}
& A=1 / 3 D+(1 / 30)\left(3 \cos ^{2} \chi-1\right) E, \\
& B=5 F+\left(3 \cos ^{2} \chi-1\right) G, \\
& C=5 H+\left(3 \cos ^{2} \chi-1\right) I \text {, } \\
& D=f_{e}^{2} \beta \mathbf{R}^{\dagger(1)} \mu \text {, } \\
& E=f_{e}^{2}\left\{\beta^{2}\left[\left(\mathrm{~m}^{\dagger} \boldsymbol{\mu}\right)^{2}-\mu^{2}\right]+3 \beta\left(\alpha_{m}-\alpha\right)\right. \\
& \left.-\beta\left(2 \mathrm{R}^{\dagger(1)}-3 \mathrm{R}^{\dagger(2)}\right) \mu\right\}, \\
& F=f_{e}^{2}\left[\beta\left(\boldsymbol{\mu}^{\dagger} \Delta \boldsymbol{\mu}^{\dagger}\right)+3 / 2 \Delta \bar{\alpha}+\mathbf{R}^{\dagger(1)} \Delta \boldsymbol{\mu}\right], \\
& G=f_{e}^{2}\left\{\beta \left[3\left(\mathbf{m}^{\dagger} \boldsymbol{\mu}\right)\left(\mathbf{m}^{\dagger} \Delta \boldsymbol{\mu}\right)-\left(\boldsymbol{\mu}^{\dagger} \Delta \boldsymbol{\mu}\right)\right.\right. \\
& \left.+3 / 2\left(\alpha_{m}-\Delta \bar{\alpha}\right)-1 / 2\left(2 \mathbf{R}^{\dagger(1)}-3 \mathbf{R}^{\dagger(2)}\right) \Delta \mu\right\}, \\
& H=f_{e}{ }^{2}(\Delta \mu)^{2} \text {, } \\
& I=f_{e}^{2}\left[3\left(\mathrm{~m}^{\dagger} \Delta \mu\right)^{2}-(\Delta \mu)^{2}\right],
\end{aligned}
$$

where $\mu^{\dagger}$ is a column vector, $\mu$ is a row vector, $\beta$ is $k^{-1} T^{-1}, h$ is Planck's constant, and $c$ is the speed of light. $\mu, S, \Delta \mu$, and $\Delta \bar{\alpha}$ are approximately the ground state electric dipole moment, the average polarizability of the solute molecule, and the changes of these quantities on excitation, respectively. $\alpha_{m}$ is the polarizability in the direction of the transition moment. The vectors $\mathbf{R}^{(1)}$ and $\mathbf{R}^{(2)}$ describe the first order effect of the external electric field on the transition moment. Deltas indicate the change of the associated quantities from the ground to the excited state. The terms involving differences of $\mathbf{R}^{(1)}$ and $\mathbf{R}^{(2)}$ and differences in polarizabilities are negligibly small in most cases. ${ }^{7}$

By determination of $L_{x}{ }^{\prime}\left(\nu_{u}\right)$ at different values of $\chi$, the values of the constants $D$ through $I$ may be obtained. The relationships between these quantities and the molecular properties of which they are a function has been thoroughly noted in Liptay. ${ }^{7}$ For the purpose of this work, it is sufficient to note that for the case where the directions of the transition moment, the ground state electric dipole moment and the excited state electric dipole moment are parallel, that $I=2 H$ and $G \approx 2 F$.

The quantities given in the equations of the constants are only approximations to the true molecular quantities which are given by

$$
\begin{aligned}
& \boldsymbol{\mu}=\left(1-f \boldsymbol{\alpha}_{g}\right)^{-1} \boldsymbol{\mu}_{g}, \\
& \alpha=\left(1-f \alpha_{\theta}\right)^{-1} \alpha_{\theta}, \\
& \Delta \mu=\left(1-f^{\prime} \alpha_{a}\right)^{-1}\left(1-f \alpha_{g}\right)^{-1}\left(1-f^{\prime} \alpha_{\theta}\right) \\
& \times\left[\mu_{a}-\left(1-f \boldsymbol{\alpha}_{g}\right)^{-1}\left(1-f \boldsymbol{\alpha}_{a}\right) \boldsymbol{\mu}_{0}\right], \\
& \Delta \alpha=\left(1-f^{\prime} \alpha_{a}\right)^{-1}\left(1-f \alpha_{g}\right)^{-1}\left\{\alpha_{a}-\alpha_{g}\right. \\
& \left.+\left(f-f^{\prime}\right) \boldsymbol{\alpha}_{\theta}\left[\alpha_{a}-\alpha_{\theta}\left(1-f \alpha_{n}\right)^{-1}\left(1-f \alpha_{a}\right)\right]\right\} .
\end{aligned}
$$

The actual field at the molecule, $\mathbf{F}_{e}$, must be determined. It may be represented as the sum of the vacuum field $\mathbf{F}_{v}$ and the reaction field, $\mathbf{F}_{R g}{ }^{8}$

$$
\mathbf{F}_{\dot{e}}=\mathbf{F}_{v}+\mathbf{F}_{R g} \text {, }
$$


where

and

$$
\mathbf{F}_{v}=f_{e} \mathbf{F}_{a}
$$

$$
\mathrm{F}_{R_{g}}=f \mu_{g}^{\prime} \text {. }
$$

Here $\mathbf{F}_{a}$ is the external field and $\boldsymbol{\mu}_{g}$ is the sum of both the permanent and induced electric moments of the solute molecule in the ground state. $F_{v}$ would be the field at the location of the solute molecule if the molecule had no permanent electric moment and its polarizability were zero. The proportionality constant of the vacuum field to the external field is a function of the solvent dielectric constant

$$
f_{c}=3 \epsilon_{D K} /\left(2 \epsilon_{D K}+1\right)
$$

However, since molecules have a finite polarizability and frequently have electric dipole moments, the reaction field ${ }^{8}$ of the solute molecule must be considered. In the approximation of the electric dipole of the solute molecule as a point dipole in the center of a sphere of radius $a$, the proportionality constant is

$$
f=2\left(\epsilon_{D K}-1\right) / a^{3}\left(2 \epsilon_{D K}+1\right)
$$

At optical frequencies $n^{2}$, the square of the refractive index, is substituted for $\epsilon_{D K}$ and $f$ is set equal to $f^{\prime}$. For nonpolar solvents $f \alpha$ and $f^{\prime} \alpha$ may be of the magnitude 0.05 to 0.2 and are therefore not necessarily negligible. ${ }^{5}$

An independent valuation of $\mu_{g}$ may be obtained from measurements of the dielectric constants of solutions. With the term $D$, this permits valuation of the

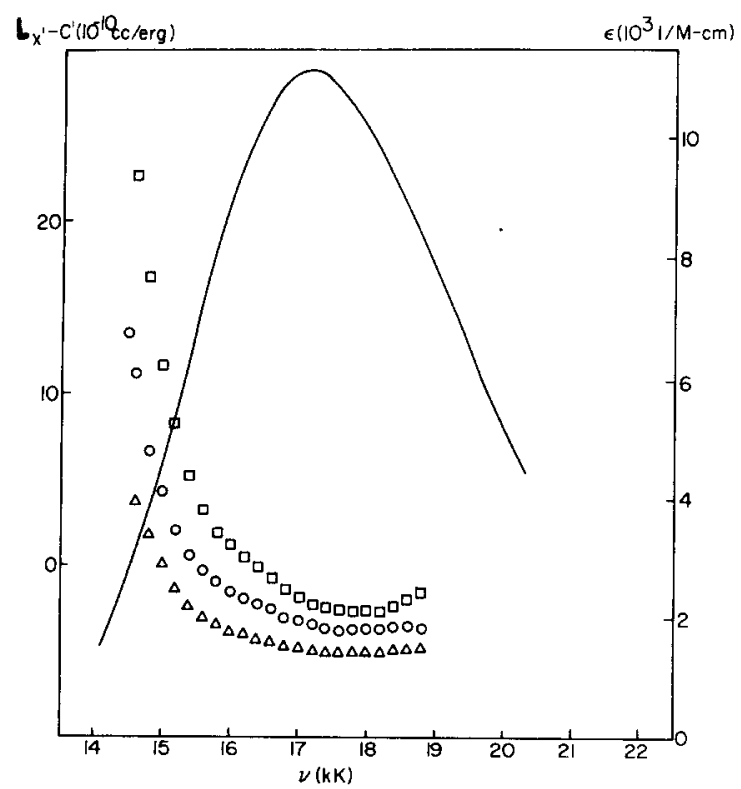

Fig. 1. The field free absorption spectrum of the charge transfer band of TCNE-stilbene is shown by the solid line. The wavenumber dependence of $L_{x}^{\prime}$ is depicted for $\chi=0^{\circ}$ (open squares), $\chi=54.7^{\circ}$ (open circles), and $\chi=90^{\circ}$ (open triangles) as determined by electro-optic measurements.

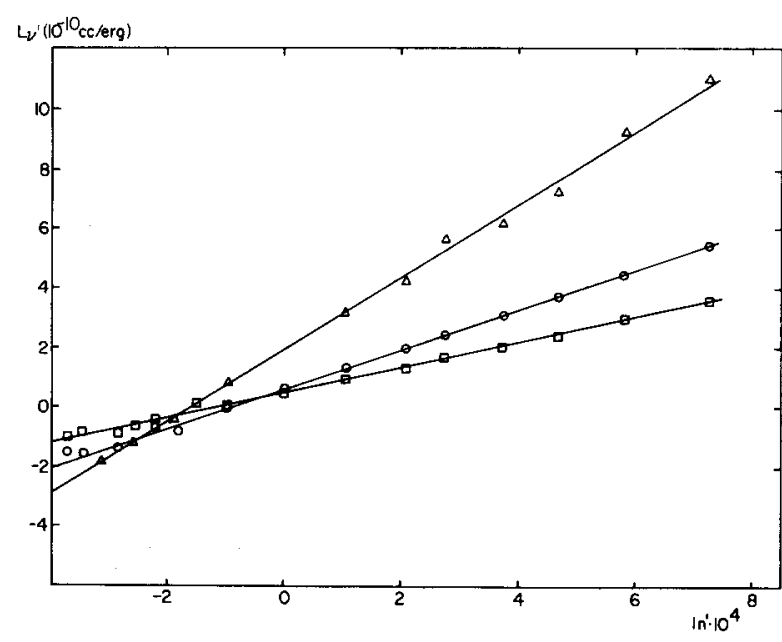

FIG. 2. The plot of $L_{\mathbf{x}}{ }^{\prime}-C_{\mathbf{x}}{ }^{\prime}$ defined in Eq. (21) against

$$
\left(\frac{d \ln (\epsilon / \nu)}{d \nu}\right)_{\nu a}
$$

for $\chi=90^{\circ}$ (open triangles), $\chi=54.7^{\circ}$ (open circles), and $\chi=0^{\circ}$ (open squares).

component of $\mathbf{R}^{(1)}$ in the direction of $\boldsymbol{\mu}_{\theta}$. If desired, the polarizability of the molecule in the ground state may also be estimated or determined independently.

\section{EXPERIMENTAL}

The experimental apparatus used for the electrochromic experiments has been described in previous papers. ${ }^{4,9}$ The external field strength of the dc field was $64 \mathrm{kV} / \mathrm{cm}$ and of the ac field was $36 \mathrm{kV} / \mathrm{cm}$. The field free absorption measurements were made with a Zeiss spectrophotometer.

The trans-stilbene of scintillation grade from Merck was used without further purification. The tetracyanoethylene (TCNE) was purified by sublimation until pure white crystals were obtained. The cyclohexane was distilled over a sodium-potassium alloy and recycled to assure both purity and dryness. Because of the very low solubility of TCNE in cyclohexane, highly concentrated stilbene solutions were used to which TCNE was added in sufficient quantity to give the desired extinction.

The equilibrium constant of the complex in cyclohexane was determined optically by the dilution procedure described by Cilento and Sanioto. ${ }^{10}$ A Beckman DU-2 spectrophotometer was used for these determinations. The value of the equilibrium constant, $K_{c}=29$, was in good agreement with the equilibrium constant of 23 obtained by Laarhoven and Nivard ${ }^{11}$ for TCNE-stilbene in methylene chloride.

The dielectric constant measurements of the solution of the complex were made on a Kahlsico Model DM 01 Dipolmeter manufactured by Firma WTW, Weilheim. Dipole moments were calculated according to the 
TABLE I. Values of quantities determined by electro-optic absorption measurements.

\begin{tabular}{lc}
\hline \hline $\boldsymbol{\nu}_{\max }(\mathbf{k K})$ & 17.2 \\
$\log \nu_{\max }$ & 4.04 \\
$D$ & $2.4 \pm 0.6 \times 10^{-10}$ \\
$E$ & $18 \pm 6 \times 10^{-10}$ \\
$F$ & $4.0 \pm 0.4 \times 10^{-22}$ \\
$G$ & $7.5 \pm 0.5 \times 10^{-22}$ \\
$H$ & $1.6 \pm 0.3 \times 10^{-34}$ \\
$I$ & $3.9 \pm 0.9 \times 10^{-34}$ \\
$\mathbf{u}_{g}{ }^{a}$ & $1.1 \pm 0.1 \mathrm{D}$ \\
$\mathbf{u}_{g}{ }^{\mathbf{b}}$ & $1.0 \pm 0.2 \mathrm{D}$ \\
$\mathbf{u}_{g}{ }^{\mathbf{c}}$ & $1.4 \pm 0.5 \mathrm{D}$ \\
$\Delta_{\mathbf{u}}$ & $10.5 \pm 0.9 \mathrm{D}$ \\
$\Delta \bar{\alpha}$ & $-5 \pm 2 \times 10^{-23}$ \\
$R_{\mu}^{(1)}$ & $6 \pm 1 \times 10^{-6}$ \\
\hline
\end{tabular}

Determined from $F$.

b Determined from $G$.

c Determined from dielectric measurements.

method developed by Briegleb and Czekalla ${ }^{12}$ for complexes. A Bausch and Lomb Abbe refractometer was used for determination of the refractive indices.

Weight measurements were made using a Mettler Gram-O-Matic semimicro single pan balance. All experiments were run at $20^{\circ} \mathrm{C}$ using a Lauda circulating water bath.

\section{RESULTS AND DISCUSSION}

The electrochromic experimental results for the TCNE-stilbene complex are shown in Fig. 1. The solid line is the field free absorption spectrum of the complex. The $L_{\chi}{ }^{\prime}$ values are shown for $\chi=90^{\circ}, 54.7^{\circ}$, and $0^{\circ}$, respectively. In Fig. 2 the plot of

$$
\begin{aligned}
L_{\chi}{ }^{\prime}-C_{\chi}{ }^{\prime}=L_{\chi}{ }^{\prime}-\left(30 h^{2} c^{2}\right)^{-1}[ & \left(\frac{d \ln (\epsilon / \nu)}{d \nu}\right)_{\nu a}^{2} \\
& \left.+\left(\frac{d^{2} \ln (\epsilon / \nu)}{d^{2} \nu}\right)_{\nu a}\right] C_{\chi}
\end{aligned}
$$

against $(d \ln (\epsilon / \nu) / d \nu)_{\nu_{a}}=\ln ^{\prime}$ is presented. The resulting straight lines indicate both the quality of the data and the fact that the frequency region studied is associated with only one electronic state.,9 The derivatives used in the determination of $A_{\chi}, B_{\chi}$, and $C_{\chi}$ from the values of $L_{x}{ }^{\prime}$ were evaluated by numerical differentiation of the absorption curve in Fig. 1. The $\chi$ dependence of $A_{x}, B_{x}$, and $C_{x}$ was then used to obtain the constants $D$ through $I$ which are given in Table I.

It can be seen that $I$ is approximately twice the value of $H$ and that $G$ is approximately twice the value of $F$. This indicates that, within error, the directions of the transition moment, the ground state electric dipole moment, and the excited state electric dipole moment are parallel for the TCNE-stilbene complex. From the Mulliken theory for molecular complexes, this is what is to be expected.
Additionally, the theory predicts that the transition moment should be polarized along the direction of the line joining the centers of the two molecules. To make any further evaluations, the dielectric constant of the solvent must be found in order to evaluate $f_{e}$. Because the stilbene in these experiments was present in much greater concentration than the complex and TCNE, $\epsilon_{D K}$ will be better approximated by the dielectric constant of the stilbene-cyclohexane solutions. Dielectric constant measurements of stilbene solutions of the concentration used in the electrochromic experiments were used in the equation for $f_{e}$; the value observed was 2.03 .

From $H$ and $I$ and taking $\mu$ and $\Delta \mu$ to be parallel, the magnitude of the change of the electric dipole moment going from the ground to the excited state can be obtained. $\Delta \mu$ is found to be $10.5 \pm 0.9 \mathrm{D}$. Since the quantity is positive, this means the electric dipole moment increases by that amount over the ground state value.

The value of the approximate ground state electric dipole moment can be obtained from $F$ using the approximation that $\mathrm{R}^{(1)}$ and $\Delta \overline{\boldsymbol{\alpha}}$ can be neglected. This gives $\mu_{\theta}=1.1 \pm 0.1 \mathrm{D}$. Similarly $\mu_{g}$ may be obtained from $G$ by neglecting the terms containing the changes in polarizabilities and the difference $\left(R^{(1)}-R^{(2)}\right)$. This gives a $\mu_{g}=1.0 \pm 0.2 \mathrm{D}$ which is in good agreement with the value obtained from the $F$ term.

These values may be compared to that obtained from the independent dielectric constant measurements. Those measurements gave $\boldsymbol{\mu}_{g}=1.4 \pm 0.5 \mathrm{D}$. Thus the values are in agreement within the limits of error. Consideration of the relations (12) and (20) indicates that the approximate values obtained for $\mu_{a}$ and $\mu_{g}$ should be higher than that for the true electric dipole moment. Since the values are the same within error, the product $f \alpha$ may be expected to be small. This is not surprising, since the cavity of diameter $a$ must be extremely large to accommodate the complex. Using $a=13 \AA$, (the approximate length of trans-stilbene) and $\alpha=10^{-22}$, it is found that $f=0.02$. Thus, there will be no significant difference between the values of the quantities directly determined from Eqs. (6) through (11) and those obtained from Eqs. (12) through (15).

From the average value of $1.2 \pm 0.3 \mathrm{D}$, obtained from all three electric dipole moment values, the value of $R_{\mu}{ }^{(1)}$ in the direction of the electric dipole can be obtained from the $D$ equation. This substitution yields a value of $6 \pm 1 \times 10^{-6}$ for $R_{\mu}^{(1)}$. If this value is resubstituted in $F$, the change of average polarizability upon excitation can be obtained: $\Delta \bar{\alpha}=-5 \pm 2 \times 10^{-23}$, thereby confirming that the change in the polarizability is small.

In the Mulliken theory, the ground state of a molecular complex may be described by a wavefunction

$$
\mathbf{\Psi}_{N}=a \psi_{0}(A, D)+b \psi_{1}\left(A^{-}-D^{+}\right)
$$

and the excited state may be described by

$$
\mathbf{\Psi}_{E}=a^{*} \psi_{1}\left(A^{-}-D^{+}\right)-b^{*} \psi_{0}(A, D)
$$


where $\psi_{0}$ is the "no-bond" wavefunction describing structures resulting from dipole-dipole and dipoleinduced dipole interactions, dispersion forces and hydrogen bonding. $\psi_{1}$ corresponds to the so-called dative structure where an electron has been completely transfered from the donor to the acceptor. The constants $a, b, a^{*}$, and $b^{*}$ may be and usually are different. Normalization of $\mathbf{\Psi}_{N}, \mathbf{\Psi}_{E}, \psi_{0}$ and $\psi_{1}$ gives:

$$
a^{2}+2 a b S+b^{2}=1
$$

and

$$
a^{* 2}+2 a^{*} b^{*} S+b^{* 2}=1
$$

where

$$
S=\left\langle\psi_{0} \mid \psi_{1}\right\rangle
$$

The squares of the coefficients represent the percentage weight of each contributing structure. Using the expression for the electric dipole moment

$$
\boldsymbol{\mu}_{N}=e\left\langle\boldsymbol{\Psi}_{N}\left|\sum \mathbf{r}_{i}\right| \mathbf{\Psi}_{N}\right\rangle
$$

and the equations (22) through (26) one finds

$$
\boldsymbol{\mu}_{N}=\boldsymbol{\mu}_{1}\left(b^{2}+a b S\right)
$$

and similarly for the excited state

$$
\mu_{E}=\mu_{1}\left(a^{* 2}-a^{*} b^{*} S\right)
$$

Therefore, with knowledge of the electric dipole moments for the ground and excited states, the coefficients of the respective wavefunctions can be obtained. From the coefficients, together with an estimate of the overlap $S$ and $\mu_{1}$, the per cent ionic character of the states may be obtained. Since $\mu_{1}$ is described by $\psi_{1}$, the charge separation in the dative state gives the main contribution to this dipole moment; $\mu_{1}=e r$ where $\mathbf{r}$ is the average distance between the charge on the donor and acceptor molecules. The sum of van der Waals distances of stilbene and TCNE is $3.4 \AA$, assuming the molecules are coplanar. This leads to $\mu_{1} \approx 16 \mathrm{D}$. $S=0.1$ may be assumed for the overlap of $b \pi-a \pi$ complexes. $^{13}$ This leads to values of $a=0.97$ and $b=0.23$ for the ground state of the TCNE-stilbene complex and $a^{*}=$ 0.87 and $b^{*}=0.49$ for the excited state. Thus, the ground state of the TCNE-stilbene complex has only $5 \%$ ionic character while its excited state has $76 \%$ ionic character.

\section{CONCLUSION}

The electrochromic effect yields values for the ground and excited state electric dipole moments of the TCNEstilbene pi molecular complex. In addition, these two moments are found to be parallel to the transition moment. As expected, the total electric dipole moment of the excited state is large having a value of $\mu_{E}=$ $12 \pm 1 \mathrm{D}$. The averaged ground state electric dipole moment of $1.1 \pm 0.2 \mathrm{D}$ agrees within experimental error with the solution dielectric constant measurement of $1.4 \pm 0.5 \mathrm{D}$. This agreement of values further indicates that the solvent cavity in which the complex is located is quite large and approximately spherical. The measurement also indicates the change of polarizability on transition from the ground to the excited state is small and negative.

The experimental data has given direct determination of the per cent ionic character of the ground and excited states of the complex. The excited state is found to be strongly ionic in character as is expected from the Mulliken theory. The charge transfer band of the TCNE-stilbene complex was also shown to arise from a single electronic transition. Experiments are being initiated on the solvatochromism ${ }^{5}$ of this complex as well as the polarized electronic spectra of TCNEstilbene single crystals.

\section{ACKNOWLEDGMENTS}

The author wishes to thank Professor W. Liptay for providing both the facilities and helpful discussions which made this research possible. Assistance with preliminary data analysis by Dr. W. Bauman and Mr. Lang and Mr. Merlet and the use of computer programs written by Mr. Wehning and Mr. Schlosser is gratefully acknowledged. Generous support of this research was provided by the Deutscher Akademischer Austauschdienst, the Petroleum Research Fund of the American Chemical Society (\#1563-G2), and the University of Nebraska Research Council.

${ }^{1}$ R. S. Mulliken, J. Am. Chem. Soc. 64, 811 (1952); J. Phys. Chem. 56, 801 (1952).

${ }^{2}$ W. Kuhn, H. Duhrkop, and H. Martin, Z. Physik. Chem. B45, 121 (1940).

${ }^{3}$ H. Labhart, Chimia (Aarau) 15, 20 (1961).

${ }^{4} \mathrm{~J}$. Czekalla and G. Wick, Ber. Bunsenges. Physik. Chem. 65, 727 (1971).

5 W. Liptay, Modern Quantum Chemistry Vol. III (Academic Press, New York, 1965), edited by O. Sinanoğlu, p. 45.; Angew. Chem. Internat. Ed. 8, 177 (1969); W. Liptay, B. Dumbacher, and $H$. Weisenberger, $Z$. Naturforsch. 23a, 1601 (1968).

${ }^{6} \mathrm{~W}$. Baumann, doctoral thesis, University of Mainz, 1970.

${ }^{7}$ W. Liptay, Z. Naturforsch. 20a, 272 (1965).

${ }^{8}$ L. Onsager, J. Am. Chem. Soc. 58, 1486 (1936).

${ }^{9} \mathrm{~W}$. Liptay, W. Eberlein, H. Weidenberg, and O. Elflein, Ber. Bunsenges. Physik. Chem. 71, 548 (1967).

${ }^{10} \mathrm{G}$. Cilento and D. L. Sanioto, Z. Physik. Chem. (Leipzig) 223, 333 (1963).

"W. H. Laarhoven and R. J. F. Nivard, Rec. Trav. Chim. Pays-Bas Belg. 70, 499 (1951).

${ }_{12}$ G. Briegleb and J. Czekalla, Z. Elektrochem. 58, 249 (1954).

13 R. S. Mulliken and W. B. Person, Molecular Complexes (Wiley-Interscience, New York, 1969), p. 127. 